\title{
QUADRATIC INEQUALITIES AND A CHARACTERIZATION OF INNER PRODUCT
}

\author{
JANUSZ MATKOWSKI
}

Abstract. Let $X$ be a real linear space and let $a \in \mathbb{R}, a \neq 0$, be fixed. Assuming that the functions $g, h: X \rightarrow \mathbb{R}$ satisfy the inequalities $g(a x+y)+h(x-a y) \leqslant a^{2} g(x)+g(y)+h(x)+$ $a^{2} h(y)$ for all $x, y \in X$, and some subhomogeneity type conditions, we prove that $h=g$, the function $g$ is a quadratic functional, and there exists a unique symmetric biadditive function $S: X^{2} \rightarrow \mathbb{R}$ such that $g(x)=S(x, x)$ for all $x \in X$.

A motivation in the theory of orthogonal additive functions is presented.

Mathematics subject classification (2010): Primary 46C15, 26D15, 39B22; Secondary 39B55.

Keywords and phrases: parallelogram equality, inner product, quadratic functional, quadratic inequality, Jordan-von Neumann theorem.

\section{REFERENCES}

[1] J. ACZÉL \& J. Dhombres, Functional equations in several variables, Encyclopedia of Mathematics and its Applications, Cambridge University Press, Cambridge-New York-New Rochelle-MelbourneSydney, 1989.

[2] D. AMIR, Characterization of inner product spaces, Birkhäuser Verlag, Basel-Boston-Stuttgart, 1986.

[3] M. M. DAY, Some characterizations of inner product spaces, Trans. Amer. Math. Soc. 62 (1947), 320 337.

[4] P. Jordan, J. von Neumann, On inner products in linear metric spaces, Ann. Math. 16 (1935), 719-723.

[5] L. SzÉKelYhidi, Convolution Type Functional Equations on Topological Abelian Groups, World Scientific Publishing Co. Inc., Teaneck, NJ, 1991. 